\title{
Real-time measurement of the emergence of superconducting order in a high-temperature superconductor
}

\author{
I. Madan, ${ }^{1,2,{ }^{*}}$ P. Kusar, ${ }^{1}$ V. V. Baranov,,${ }^{1,3}$ M. Lu-Dac, ${ }^{1}$ V. V. Kabanov,${ }^{1}$ T. Mertelj ${ }^{1,4}$ and D. Mihailovic ${ }^{1,2,4}$ \\ ${ }^{1}$ Complex Matter Department, Jozef Stefan Institute, Jamova 39, 1000 Ljubljana, Slovenia \\ ${ }^{2}$ Jozef Stefan International Postgraduate School, Jamova 39, 1000 Ljubljana, Slovenia \\ ${ }^{3}$ Department of Physics, University of Antwerp, Groenenborgerlaan 171, 2020 Antwerp, Belgium \\ ${ }^{4}$ CENN Nanocentre, Jamova 39, 1000 Ljubljana, Slovenia
}

(Received 17 January 2016; revised manuscript received 4 June 2016; published 30 June 2016)

\begin{abstract}
Systems which rapidly evolve through symmetry-breaking transitions on timescales comparable to the fluctuation timescale of the single-particle excitations may behave very differently than under controlled near-ergodic conditions. A real-time investigation with high temporal resolution may reveal insights into the ordering through the transition that are not available in static experiments. We present an investigation of the system trajectory through a normal-to-superconductor transition in a prototype high-temperature superconducting cuprate in which such a situation occurs. Using a multiple pulse femtosecond spectroscopy technique we measure the system trajectory and time evolution of the single-particle excitations through the transition in $\mathrm{La}_{1.9} \mathrm{Sr}_{0.1} \mathrm{CuO}_{4}$ and compare the data to a simulation based on the time-dependent Ginzburg-Landau theory, using the laser excitation fluence as an adjustable parameter controlling the quench conditions in both experiment and theory. The comparison reveals the presence of significant superconducting fluctuations which precede the transition on short timescales. By including superconducting fluctuations as a seed for the growth of the superconducting order we can obtain a satisfactory agreement of the theory with the experiment. Remarkably, the pseudogap excitations apparently play no role in this process.
\end{abstract}

DOI: 10.1103/PhysRevB.93.224520

\section{INTRODUCTION}

The study of the time evolution of complex systems through symmetry breaking transitions (SBT) is of great fundamental interest in different areas of physics [1-3]. An SBT of particular general interest is the normal-to-superconducting $(N \rightarrow S)$ state transition in which a Lorentz noninvariant system breaks gauge invariance [4]. By studying the $N \rightarrow S$ transition in time-evolving systems, rather than by slowly varying the temperature through the transition, one can in principle gain information on the dynamical behavior of elementary excitations which lead to the formation of a superconducting condensate and the collective ordering behavior, leading to insights into nonergodic phenomena of collectively ordered systems as well as the mechanism of superconductivity. Particularly, ergodicity breaking in rapidly evolving systems leads to the appearance of topological defects (vortices).

A description of the dynamical behavior of the gauge noninvariant systems is often discussed in terms of time-dependent Ginzburg-Landau theory (TDGL theory). It has been initially applied to the problem of nonequilibrium phase transitions by Kibble and Zurek who considered the appearance of topological defects accompanying the transition [5,6]. The Kibble-Zurek description has been indirectly confirmed to be appropriate by static experiments in which trapped vortices were studied [7,8]. In this paper, beyond previous static studies, we study real-time evolution of the superconducting order in the nonequilibrium phase transition. We investigate the applicability of the TDGL theory to the phase transition

\footnotetext{
*Corresponding author: ivan.madan@ijs.si
}

problem and provide a minimal formulation sufficient to describe the data.

The paper is organized in the following way: We first introduce the problem of a nonhomogeneous nonequilibrium phase transition and overview previous ultrafast studies of the condensate dynamics in cuprates. We then introduce the three-pulse technique and describe the data. In the second half of the paper we present numerical simulations of the $S \rightarrow N$ and $N \rightarrow S$ transitions with the TDGL theory, with the aim of reproducing the data as faithfully as possible with the minimal set of adjustable parameters.

\section{LASER INDUCED NONEQUILIBRIUM PHASE TRANSITIONS IN SUPERCONDUCTORS}

To achieve nonequilibrium conditions we require that the inverse of the cooling rate, the quench time $\tau_{\mathrm{q}}$, be comparable to the intrinsic collective system relaxation time $\tau_{\mathrm{GL}}=$ $\pi \hbar / 8 k\left(T-T_{\mathrm{c}}\right) \simeq 10^{-13}-10^{-12}$ s [3,9-11]. In experiments reported so far, the quench was physically limited to the $n s$ timescale either by heat diffusion processes or duration of the optical pulse used for driving the transition [8].

With femtosecond optical spectroscopy, the nonequilibrium regime of the phase transition, and particularly the critical region on the timescale of $\tau_{\mathrm{GL}}$, become accessible in real time. By properly adjusting the optical pulse energy, the limitations on the quench time set by heat diffusion processes can be overcome: For moderate fluences the electronic subsystem gets highly perturbed [12-14] while the lattice remains only weakly excited. In this case the cooling rate is defined by the energy exchange between the electronic and lattice subsystems, which typically occurs on the sub-ps timescale [15], which is much faster than heat diffusion. 
The dynamics of the superconducting condensate has been previously investigated by various ultrafast techniques, originally in the low-perturbation regime and more recently completely suppressing the condensate (See Ref. [16] for extensive review). Optical investigations have shown that at low excitation the dynamics of the condensate is characterized by the Rothwarf-Taylor bottleneck processes [17]. In this regime the relaxation timescale is given by either the high-energy phonon escape or the anharmonic decay time. For this regime it is typical that the relaxation rate is proportional to the pump fluence at low temperatures $[18,19]$. Because the recombination rate depends on the density of quasiparticles, at low excitation densities it might become smaller than inverse quasiparticle diffusion time, so the latter process may become significant [20].

At higher pump fluences the photoinduced $S \rightarrow N$ transition is induced. For cuprates it was initially observed in optics [12] and has been confirmed by $\mathrm{THz}$ studies [21,22]. The systematic fluence dependence studies of the recovery of the $S$ gap in the vicinity of $S \rightarrow N$ transition were performed on BSCCO by ARPES [18] where it was found that the gap recovery rate is momentum independent, indicating applicability of the concept of a nonequilibrium order parameter. While ARPES directly measures the gap evolution in BSCCO, the characteristic timescale of the process is very close to that of the electronic temperature evolution given by the three temperature model [23], thus making it hard to determine the mechanism responsible for the dynamics. The recovery of the superconducting order in LSCO studied in this paper occurs on the timescale which is an order of magnitude larger than the electronic temperature equilibration processes time, which allows us to monitor the nonequilibrium dynamics of the order parameter.

Optical experiments are intrinsically inhomogeneous due to a finite light penetration depth $\lambda_{p}$. This affects not only the data analysis but also the physics of the transition. Due to the exponential depth distribution of the absorbed energy, the superconducting condensate is destroyed only up to a certain depth. This results in a sharp boundary between the $N$ and $S$ states. After the quench the boundary propagates towards the surface and is expected to reach it on a timescale $\tau_{\psi} \sim$ $\lambda_{\mathrm{p}} / v_{\psi} \sim \lambda_{\mathrm{p}} \tau_{\mathrm{GL}} / \xi_{\perp} \sim 10^{3} \tau_{\mathrm{GL}}$, where $v_{\psi}$ is the velocity of the $S / N$ boundary [24] and $\xi_{\perp}$ is the out-of-plane $S$ coherence length. Though the boundary propagation is relatively slow compared to $\tau_{\mathrm{GL}}$, the physics of the transition depends on how it relates to the propagation of the temperature front, which is defined by the quench conditions. Two regimes are possible: The temperature front propagation velocity $v_{\mathrm{T}}$ can be either larger (rapid quench) or smaller (slow quench) than the characteristic critical value $v_{\text {crit }} \approx v_{\psi} \sqrt[4]{\tau_{\mathrm{GL}} / \tau_{\mathrm{q}}} \sim 10^{5} \mathrm{~cm} / \mathrm{s}$ [5]. In the rapid quench limit when $v_{\mathrm{T}}>v_{\text {crit }}$, the normal region between the temperature front and $S / N$ boundary is supercooled and the order parameter grows from fluctuations. In this case one can expect vortex formation according to the Kibble-Zurek (KZ) mechanism. In the slow quench limit $\left(v_{\mathrm{T}}<v_{\text {crit }}\right)$ the condensate forms instantaneously in the wake of the temperature front so that the phase of the order parameter is defined by the bulk value and vortex formation becomes suppressed [5,25].

As we shall see, both cases are accessible in our experiments by changing the laser fluence: At low fluences, only the (a)
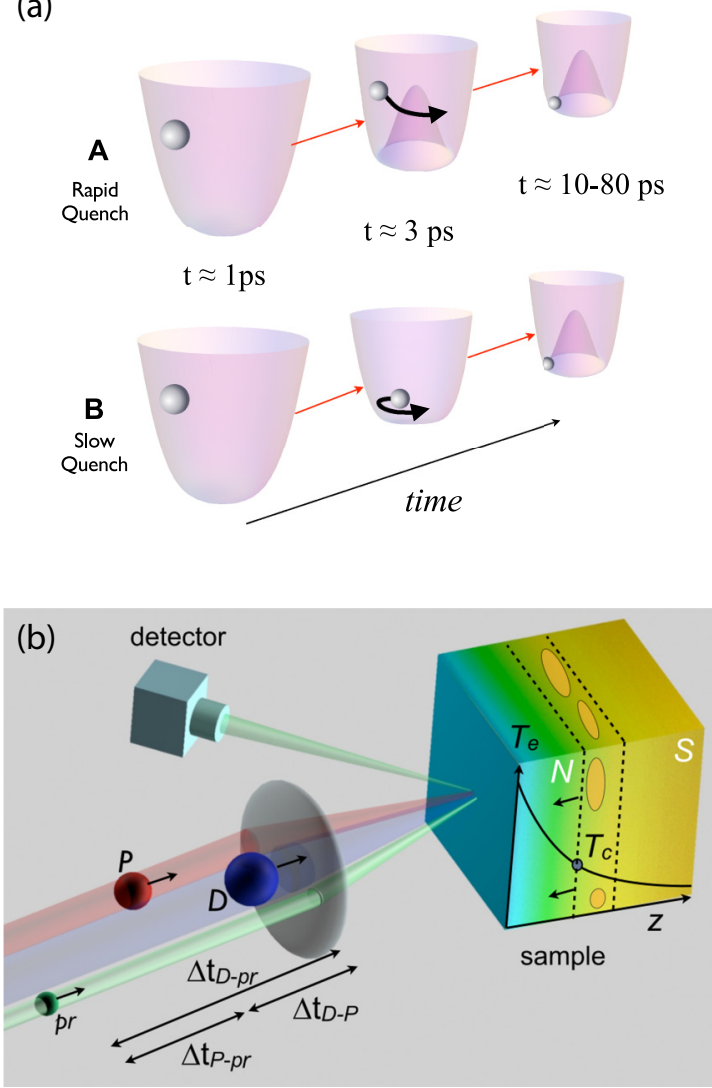

FIG. 1. (a) The system trajectory (depicted by the silver ball) in a temporally evolving potential. In the rapid quench scenario (A), the potential changes faster than the system can follow. The opposite is true in the slow quench scenario (B). (b) A schematic diagram of the pulse sequence. The time delays $\Delta t_{\mathrm{D}-\mathrm{P}}, \Delta t_{\mathrm{D}-\mathrm{pr}}$ and $\Delta t_{\mathrm{P}-\mathrm{pr}}$ refer to delays between the $D, P$ and $p r$ pulses depicted in blue, red and green respectively. The $S / N$ phase boundary moves with velocity $v_{\psi}$ towards the surface. Vortices are created in the wake of the temperature front whose position is given by $T(r, t)=T_{\mathrm{c}}$.

electrons are heated above $T_{\mathrm{c}}$. They cool rapidly through $T_{\mathrm{c}}$, so the quench rate $\gamma_{\mathrm{q}}=(d T / d t) / T_{\mathrm{c}}$ is fast [15]. With large fluences, the lattice is heated above $T_{\mathrm{c}}$. Its cooling is defined by the heat diffusion so the quench rate is much slower.

\section{EXPERIMENTAL CONSIDERATIONS}

To measure the trajectory of the system through the $N \rightarrow S$ transition, we use a three pulse technique shown schematically in Fig. 1(b). The first destruction (D) laser pulse strongly perturbs the electronic subsystem initiating the $S \rightarrow N$ transition on a timescale of $\sim 0.5-0.8$ ps [12]. The recovery of the $S$ state in the ensuing $N \rightarrow S$ transition is measured by means of pump-probe $(P-p r)$ transient reflectivity $\Delta R\left(\Delta t_{\mathrm{P}-\mathrm{pr}}\right) / R$ measurements. The pump-probe response is recorded at a set of delays $\Delta t_{\mathrm{D}-\mathrm{P}}$ between $D$ and $P$ pulses [26]. For each value of the $\Delta t_{\mathrm{D}-\mathrm{P}}$ delay the amplitude of the response $A_{\mathrm{S}}=(\Delta R / R)_{\mathrm{S}}\left(\Delta t_{\mathrm{P}-\mathrm{pr}}^{\mathrm{read}}\right)$ is extracted, and, when plotted as a function of $\Delta t_{\mathrm{D}-\mathrm{P}}$, is a measure of the trajectory of the system (see Supplemental Material [27] for the details of the amplitude extraction). It is then compared to the modeled behavior of the order parameter $\psi(t)$ using an appropriate response function. 

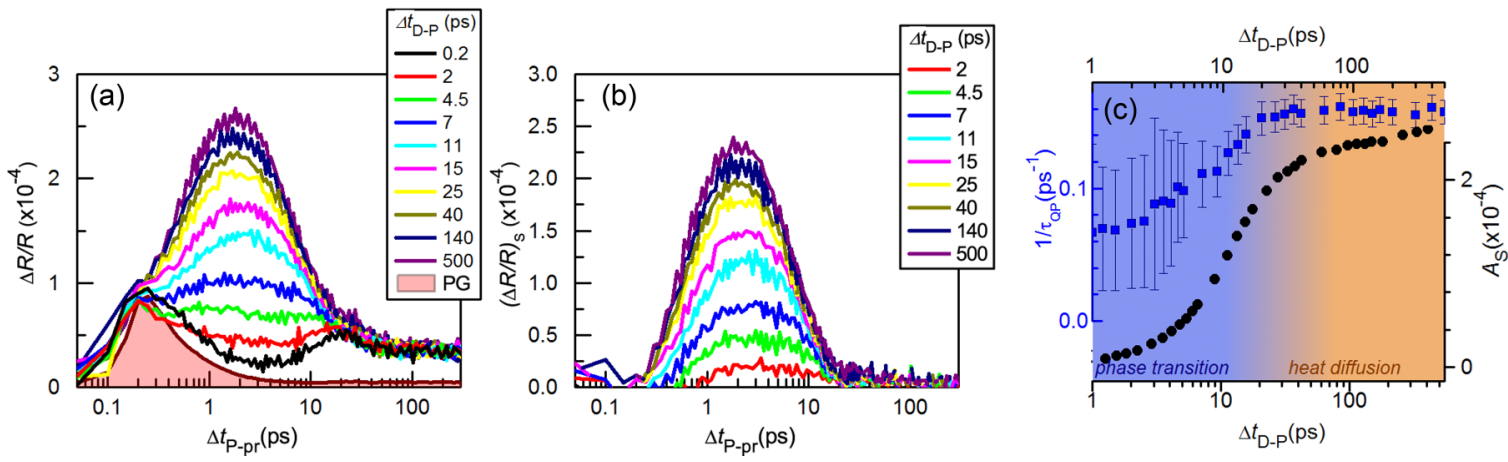

FIG. 2. (a) The transient reflectivity $\Delta R / R$ for $\mathrm{La}_{1.9} \mathrm{Sr}_{0.1} \mathrm{CuO}_{4}$ at $4 \mathrm{~K}$ as a function of $\Delta t_{\mathrm{P}-\mathrm{pr}}$, for different $\Delta t_{\mathrm{D}-\mathrm{P}}$. The $\mathrm{D}$ pulse fluence is $\mathcal{F}_{\mathrm{D}}=12 \mu \mathrm{J} / \mathrm{cm}^{2}$, which is approximately three times above the destruction threshold $\left(\mathcal{F}_{\mathrm{T}} \simeq 4.2 \mu \mathrm{J} / \mathrm{cm}^{2}\right)[12]$. The red shaded curve indicates the pseudogap signal measured above $T_{\mathrm{c}}$. (b) Superconducting component extracted from data in (a) by subtracting $\Delta t_{\mathrm{D}-\mathrm{P}}=0.2 \mathrm{ps}$ line. This dataset is used to extract the evolution of the initial quasiparticle decay rate. (c) Black squares-the amplitude of the superconducting component $A_{\mathrm{S}}$ extracted from (a) after subtraction of the PG. The blue squares are $1 / \tau_{\mathrm{QP}}$ as a function of $\Delta t_{\mathrm{D}-\mathrm{P}}$. The recovery of the system is schematically divided into phase transition region (blue background) where order parameter is not thermal, and into the thermal diffusion (orange background) where the transition is effectively over and order parameter is defined solely by the temperature.

The change of the reflectivity at optical frequencies may contain various contributions, such as a Drude contribution due to photoinduced changes of the plasma frequency and scattering rate, contributions from interband transitions and a superconducting contribution from the Mattis-Bardeen term [16]. The dynamics in a two-pulse experiment is the sum of all such contributions and can vary significantly with the probe wavelength $[28,29]$, and consequently the quasiparticle relaxation rate measured by two-pulse optical experiments might be different from the order parameter dynamics [30,31]. Nevertheless, we can clearly distinguish between nonsuperconducting and superconducting contributions from the fluence and temperature dependence of the data. Moreover, experimentally it was determined that in the material investigated here the amplitude of the superconducting component of the transient optical reflectivity is proportional to the optical conductivity in the $\mathrm{THz}$ regime [22]. The latter is dominated by the inductive contribution of the condensate, so we can relate $(\Delta R / R)_{\mathrm{s}}$ to the superfluid density. In the present paper we therefore use a phenomenological approach to the response function, which is based on the only assumption that the maximum value of the transient reflectivity can be presented as a unique function of the order parameter $(\Delta R / R)_{\mathrm{s}}^{\max }=A_{\mathrm{s}}(\psi)$. This is further justified by the observation that the temperature dependence of the response is identical for various excitation conditions [12]. The actual shape of $A_{\mathrm{s}}(\psi)$ (Fig. S1) is established from the temperature dependence of the pump-probe response. (See Supplemental Material [27] for a rigorous discussion of the response.)

The measurements were performed on a $\mathrm{La}_{1.9} \mathrm{Sr}_{0.1} \mathrm{CuO}_{4}$ (LSCO) single crystal as a prototype single-layer cuprate, which is extensively studied by the pump-probe technique [12,32]. Laser pulses from a $250 \mathrm{KHz}$ Ti:Sapphire regenerative amplifier with a central wavelength of $800 \mathrm{~nm}$ and pulse lengths of $60 \mathrm{fs}$ were used throughout. The probe polarization was perpendicular to the pump and destruction pulses. The pump and probe fluences were below $1.3 \mu \mathrm{J} / \mathrm{cm}^{2}$ to ensure linearity of the response [12]. The critical temperature of LSCO $\left(T_{\mathrm{c}}=28 \mathrm{~K}\right)$ is sufficiently high so that systematic fluence dependences can be measured, yet low enough so that the theoretical estimate of $\tau_{\mathrm{GL}}=3 /\left(T-T_{c}\right) \mathrm{ps} \approx 100 \mathrm{fs}$ is longer than our temporal resolution. The laser fluence required to destroy the superconducting state on the surface (photodestruction threshold) has been previously determined to be $\mathcal{F}_{\mathrm{T}}=4.2 \pm 1.7 \mu \mathrm{J} / \mathrm{cm}^{2}$ [12]. In the presented experiment we vary the $D$-pulse fluence from 4 to $34 \mu \mathrm{J} / \mathrm{cm}^{2}$.

\section{TRANSIENT REFLECTIVITY DATA THROUGH THE SUPERCONDUCTING TRANSITION}

A representative dataset obtained in a three-pulse experiment is shown in Fig. 2(a). It depicts the normalized transient reflectivity $\Delta R\left(\Delta t_{\mathrm{P}-\mathrm{pr}}\right) / R$ traces for different $\Delta t_{\mathrm{D}-\mathrm{P}}$ delays during the system recovery measured at $4 \mathrm{~K}$ with $D$ pulse fluence $\mathcal{F}_{\mathrm{D}}=12 \mu \mathrm{J} / \mathrm{cm}^{2}$. Two distinct and easily identifiable contributions are observed: a pseudogap (PG) response $(\Delta R / R)_{\mathrm{PG}}$ which peaks around $0.2 \mathrm{ps}$, and the quasiparticle $(\mathrm{QP})$ recombination across the superconducting gap, i.e., the superconducting response $(\Delta R / R)_{\mathrm{S}}$ which peaks near $2 \mathrm{ps,}$ extending to tens of ps $[32,33]$. This simultaneous appearance of the pseudogap and superconducting contributions below $T_{\mathrm{c}}$ is common for most of the cuprates $[28,32,34,35]$. The former is characterized by a subpicosecond weakly temperature dependent relaxation, while the dynamics of the latter can strongly vary from a few ps to tens of picoseconds. These optical dynamical features correspond to the dynamics observed in the mid-IR and $\mathrm{THz}$ regime $[31,36]$, although some details can be different at optical response frequencies. The pseudogap and superconducting contributions can be distinguished not only by their temperature and dynamical signatures, but more clearly in the multicolor experiments $[28,37]$ and by polarization selection rules [38]. Most importantly for our experiments - the pseudogap response in LSCO is linear with the photoexcitation fluence up to $\mathcal{F}_{\mathrm{PG}} \sim 750 \pm 200 \mu \mathrm{J} / \mathrm{cm}^{2}$ [33], which is two orders of magnitude higher than the superconducting $\mathcal{F}_{\mathrm{T}}$. Thus it is completely unaffected by the $\mathrm{D}$ pulse, which is confirmed by measurements above $T_{\mathrm{c}}$.

In Fig. 2(a) we clearly observe that $(\Delta R / R)_{\mathrm{S}}$ gradually increases with increasing delay $\Delta t_{\mathrm{D}-\mathrm{P}}$ indicating the recovery of the $\mathrm{S}$ state, while $(\Delta R / R)_{\mathrm{PG}}$ remains intact by the 


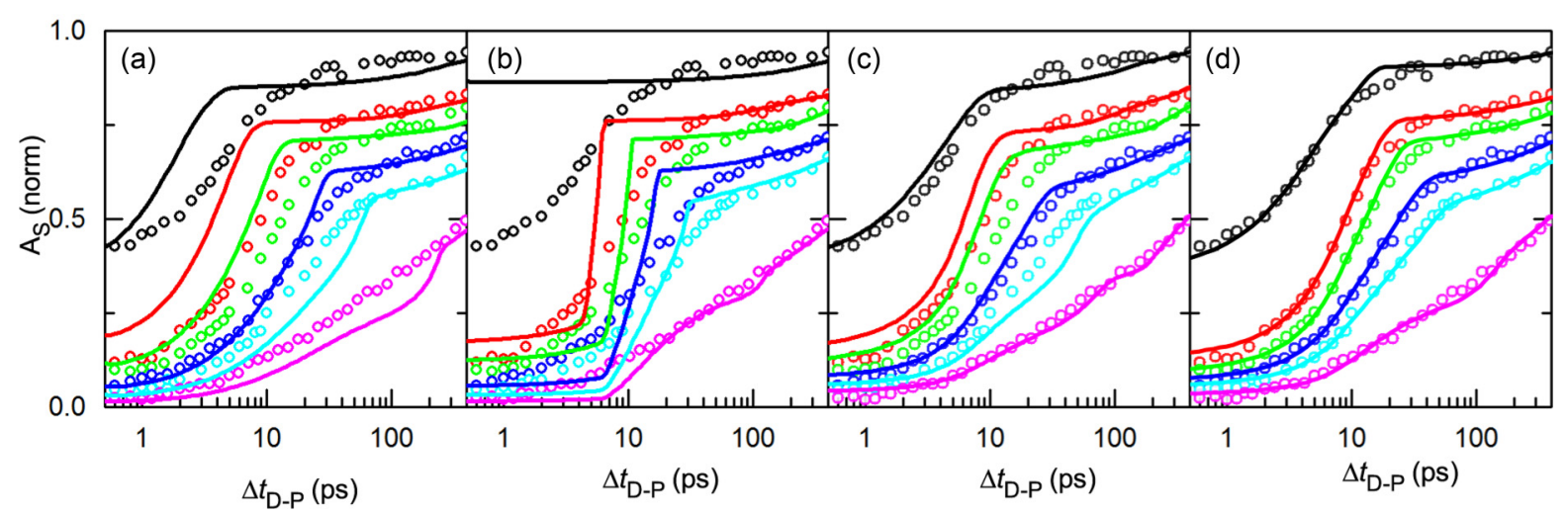

FIG. 3. Comparison of experimentally measured data [circles, values of fluence are 4 (black), 9 (red), 12 (green), 18 (blue), 24 (cyan), and 34 (magenta) $\left.\mu \mathrm{J} / \mathrm{cm}^{2}\right]$ and the calculated $A_{S}$ obtained within different formulations of the problem (solid lines): the TDGL solution with the initial conditions described by (a) $\psi(0, z)=\sqrt{1-T_{\text {bath }} / T_{\mathrm{c}}}$, (b) Eq. (3), (c) Eq. (4) (represented in Fig. 5), (d) $\psi_{\text {fluc }}(0, z)=\kappa z$.

destruction pulse and does not show any change with $\Delta t_{\mathrm{D}-\mathrm{P}}$. A distinct feature in Fig. 2(a) is an apparent convergence of all curves after $\Delta t_{\mathrm{P}-\mathrm{pr}} \sim 20 \mathrm{ps}$, which appears as a hump for small $\Delta t_{\mathrm{D}-\mathrm{P}}$ delays. There are two contributions to the signal at this delay: Firstly, the signal recovery is associated with the gap recovery itself (see also Sec. II of Supplemental Material [27]), and secondly, there is a contribution of the pump pulse to the lattice heating. This part is dominant and relaxes on the 100 ps time scale characteristic of the thermal diffusion process.

$(\Delta R / R)_{\mathrm{PG}}$ is known to be independent of $T$ at temperatures below $100 \mathrm{~K}$ in this material [32]. We are interested in the superconducting order, so for further analysis we subtract the pseudogap contribution from the data [39] and plot the extracted superconducting component in Fig. 2(b). We read out the amplitude $A_{\mathrm{S}}$ at the probe delay corresponding to the maximum of the unperturbed response and plot it as a function of $\Delta t_{\mathrm{D}-\mathrm{P}}$ in Fig. 2(c) (shown in black circles).

From the exponential fits of the initial decay of $(\Delta R / R)_{\mathrm{S}}$ $\left(\Delta t_{\mathrm{P}-\mathrm{pr}}\right)$ we obtain the $\mathrm{QP}$ relaxation time $\tau_{\mathrm{QP}}$ as a function of $\Delta t_{\mathrm{D}-\mathrm{P}}$ plotted in Fig. 2(c). We observe that $1 / \tau_{\mathrm{QP}}$ shows a similar time evolution to that of $A_{\mathrm{S}}$. If we assume that $1 / \tau_{\mathrm{QP}} \propto \Delta_{\mathrm{S}}$, where $\Delta_{\mathrm{S}}$ is the superconducting gap $[34,40]$, the observed dependence of $\tau_{\mathrm{QP}}$ on $\Delta t_{\mathrm{D}-\mathrm{P}}$ is consistent with the growth of the $\mathrm{S}$ gap with $\Delta t_{\mathrm{D}-\mathrm{P}}$. The time evolution of $A_{\mathrm{S}}$ and $\tau_{\mathrm{QP}}$ identifies the recovery of superconducting order on a $10 \mathrm{ps}$ timescale. The measured dependence of the trajectory $A_{\mathrm{S}}\left(\Delta t_{\mathrm{D}-\mathrm{P}}\right)$ for different fluences $\mathcal{F}$ is shown in Fig. 3, where it is compared to the simulated trajectories from different models described below.

Qualitatively one can see that recovery of the order parameter is slower at higher fluences, compared to low and intermediate fluences. The effect has an obvious explanation when one takes into account lattice heating and the fact that the relaxation time increases when $T \rightarrow T_{\mathrm{c}}$. This effect is expected on the basis of TDGL equations.

\section{MODELING WITH TIME-DEPENDENT GINZBURG-LANDAU THEORY}

In the following section our aim is to formulate a minimal TDGL model which captures the observed behavior using the laser pulse fluence as the only externally controlled parameter. We consider only the real part of TDGL equations, as the optical response is insensitive to the phase of the order parameter [41]. (In the Supplemental Material [27] we show the solution of the full set of TDGL equations to qualitatively account for dynamics of the phase and vortex dynamics.) The basic TDGL equation describing the order parameter $\psi(t, z)$ dynamics is [42]:

$$
\frac{\partial \psi}{\partial t}=\alpha_{r}(t, z) \psi-\psi|\psi|^{2}+\nabla^{2} \psi,
$$

where we have omitted explicit dependence of $\psi$ on $t$ and $z$, and the temporal and spatial coordinates are measured in units of $\tau_{\mathrm{GL}}$ (fitting variable) and coherence length $(\xi=0.2 \mathrm{~nm}$ [43]) at $T=0 \mathrm{~K}$, respectively. In the transient reflectivity calculation the order parameter is weighted by the probe penetration depth $\lambda_{800 \mathrm{~nm}}=150 \mathrm{~nm}$ [12].

The system is driven by the electronic temperature $T_{\mathrm{e}}$, which enters TDGL via $\alpha_{\mathrm{r}}(t, z)=\left(1-T_{\mathrm{e}}(t, z) / T_{\mathrm{c}}\right)$. The temperature is time dependent and also depends on the depth in the sample. To calculate $T_{\mathrm{e}}(t, z)$, we assume that electrons are preferentially coupled to a particular boson (phonon and/or spin excitation), which in turn releases its energy to the lattice. This three-temperature model (3TM) has been used in the past to describe the normal state ultrafast response in unconventional superconductors $[23,44]$. In principle, the 3TM describes the destruction of the condensate, defines the recovery timescales, and should also describe the slow diffusion processes which are clearly present in the data [Fig. 2(c)]. To account for the latter we introduce the thermal diffusivity $\kappa$ as a fitting parameter, which does not affect short timescales. The final set of equations from which we obtain $T_{\mathrm{e}}(t, z)$ is then:

$$
\begin{array}{r}
\gamma_{\mathrm{e}} T_{\mathrm{e}} \dot{T}_{\mathrm{e}}=-\gamma_{\mathrm{ep}}\left(T_{\mathrm{e}}-T_{\mathrm{p}}\right)+P(t) \\
C_{\mathrm{p}} \dot{T}_{\mathrm{p}}=-\gamma_{\mathrm{ep}}\left(T_{\mathrm{p}}-T_{\mathrm{e}}\right)-\gamma_{\mathrm{pl}}\left(T_{\mathrm{p}}-T_{1}\right) \\
C_{\mathrm{l}} \dot{T}_{1}=-\gamma_{\mathrm{pl}}\left(T_{1}-T_{\mathrm{p}}\right)+\kappa \frac{\partial^{2} T_{1}}{\partial z^{2}},
\end{array}
$$

where $\gamma_{\mathrm{e}}=2.5 \mathrm{~mJ} / \mathrm{mol} / \mathrm{K}^{2}$ [45] is the electronic specific heat coefficient, $\gamma_{i j}$ represents the coupling between the $i$ th and $j$ th bath, $T_{i}$ is the temperature of the corresponding system 


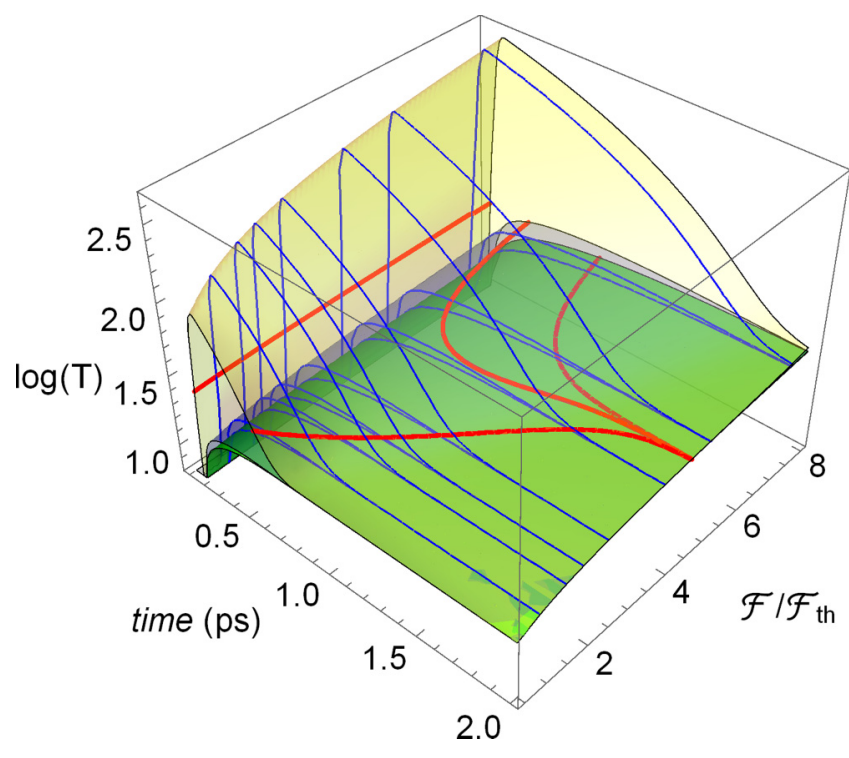

FIG. 4. Three surfaces showing the calculated time evolution of the logarithm of $T_{\mathrm{e}}$ (yellow), $T_{\mathrm{p}}$ (blue), and $T_{\mathrm{L}}$ (green) as a function of incident fluence. The initial temperature of the sample is $4 \mathrm{~K}$. $T_{\mathrm{p}}$ and $T_{\mathrm{L}}$ are very close, but $T_{\mathrm{e}}$ reaches in excess of $400 \mathrm{~K}$. The blue lines correspond to fluences used in the experiment. The red lines indicate where $T_{\mathrm{e}}, T_{\mathrm{p}}$, and $T_{\mathrm{L}}$ cross $T_{\mathrm{c}}=28 \mathrm{~K}$. $T_{\mathrm{e}}$ is used in the modeling of the order parameter. Fast quench corresponds to $\mathcal{F}<5 \mathcal{F}_{\text {th }} \simeq 23 \mu \mathrm{J} / \mathrm{cm}^{2}$.

(indices e, p, and 1 are for electronic, hot boson, and lattice, respectively), and $P(t)=\frac{\mathcal{F}}{2 \pi w} \exp \left(-t^{2} / 2 w^{2}\right) \exp \left(-z / \lambda_{800 \mathrm{~nm}}\right)$ is the optical excitation with the pulse width at half maximum given by $2 \sqrt{2 \ln 2} w=60 \mathrm{fs}$. We also assume that the total phonon heat capacity $C=C_{\mathrm{p}}+C_{\mathrm{l}}$, where $C_{\mathrm{p}}=\alpha C_{0}$ and $C_{1}=(1-\alpha) C_{0}$ is the heat capacity of the hot bosons and the lattice bath, respectively, $\alpha=0.2$ is the fraction of phonon modes which are strongly coupled to electrons [23]. The coefficients of the 3TM model $\gamma_{\mathrm{ep}}$ and $\gamma_{\mathrm{pl}}$ can be estimated using the electronic and lattice thermal constants $\gamma_{\mathrm{e}}$ and $C$, the measured electron-phonon relaxation rate $\gamma_{1}=340 \mathrm{~K} / \mathrm{ps}$ and the phonon-phonon relaxation time $\tau_{\mathrm{ph}}=0.6 \mathrm{ps}$ [15]: $\gamma_{\mathrm{ep}}=\gamma_{\mathrm{e}} \gamma_{\mathrm{l}}$ and $\gamma_{\mathrm{pl}}=C_{\mathrm{p}} / \tau_{\mathrm{ph}}$. The temperature dependence of the phonon heat capacity $C$ is obtained from published thermal data [46].

Solving Eq. (2) we obtain the time and depth dependence of $T_{\mathrm{e}}, T_{\mathrm{p}}$ and $T_{1}$. In Fig. 4 we plot the values of the corresponding temperatures on the sample surface for different fluences used in the experiments. Initially, the pulse rapidly heats the electronic system, but energy is quickly transferred to the strongly coupled bosons and the lattice on a timescale $\sim 1 \mathrm{ps,}$ whereafter the three temperatures rapidly merge. Note that this timescale is of the same order as the destruction of the $S$ state [47]. We see in Fig. 4 that for low excitation fluences $\mathcal{F} / \mathcal{F}_{\text {th }}$, the quench rate $\gamma_{\mathrm{Q}}=\left(d T_{e} / d t\right)_{T_{\mathrm{c}}}$ through $T_{\mathrm{c}}$ (red line) is fast, of the order of $4 \times 10^{14} \mathrm{~K} / \mathrm{s}$ and $T_{\mathrm{e}} \simeq T_{\mathrm{p}} \simeq T_{1}$ already after $\sim 1$ ps. With higher fluences, $>5 \mathcal{F}_{\text {th }}$ when $T_{\mathrm{p}}$ and $T_{1}$ both exceed the superconducting $T_{\mathrm{c}}$, the cooling rate is mainly determined by thermal diffusion on timescales well beyond $\sim 1$ ps. We emphasize that this crossover from a rapid to slow quench is quite general and does not rely on the specific details of the 3TM. Having calculated $T_{\mathrm{e}}(t, z)$, we are in a position to calculate $\psi(t, z)$, and $A_{\mathrm{S}}(t)$.

First, we try to describe both the destruction and recovery of the superconducting condensate within the above TDGL model. The only adjustable parameter in the fitting procedure is $\tau_{\mathrm{GL}}$ since the initial conditions are given by the equilibrium order parameter $\psi(0, z)=\sqrt{1-T_{\text {bath }} / T_{\mathrm{c}}}$. A set of simulated trajectories with the optimized $\tau_{\mathrm{GL}}=450 \mathrm{fs}$ is shown in Fig. 3(a). Despite optimization of $\tau_{\mathrm{GL}}$ the model trajectories are in poor agreement with the experimental data.

To better understand the poor agreement let us consider first in detail the destruction stage, i.e., $S \rightarrow N$ transition. The transition is driven by a temperature burst described by the $\alpha$ term of Eq. (1) where the relation between $\tau_{\mathrm{GL}}$ (the only free parameter) and the duration of the temperature burst is crucial. The condensate can follow an intense short temperature perturbation only if $\tau_{\mathrm{GL}}$ is shorter than the duration of the perturbation $\sim 1 \mathrm{ps}$. Such a short $\tau_{\mathrm{GL}}$ implies, however, also a rapid recovery, significantly faster than experimentally observed, as seen in Fig. 3(a). In the slow quench regime $\left(\mathcal{F} \geqslant 18 \mu \mathrm{J} / \mathrm{cm}^{2}\right)$ the perturbation duration is longer and the OP suppression is more effective. The OP follows the temperature evolution adiabatically resulting in a recovery on the timescale closer to the experimentally observed. On the other hand, if $\tau_{\mathrm{GL}}$ is much longer than the perturbation, the condensate cannot follow the temperature dynamics and the condensate remains undestroyed.

The TDGL equations therefore cannot provide a good description of a complete destruction-recovery process. This can be understood considering that during the destruction the electron and phonon distribution functions are clearly not thermal leading to effects beyond the TDGL and 3TM description [48].

Nevertheless, the TDGL theory is expected to provide a better description of the recovery of the condensate dynamics after the $S \rightarrow N$ transition has occurred and the temperatures have equilibrated. The state of the system after the photodestruction is then represented by initial conditions, and the corresponding solution of the TDGL equation describes the ensuing recovery dynamics, i.e., the $N \rightarrow S$ transition.

Next, we skip the modeling of the destruction process, focusing on the recovery. We begin by specifying the initial depth distribution of the condensate density. We assume that the order parameter is completely destroyed in the region of the sample where $\mathcal{F}(z)>\mathcal{F}_{\mathrm{T}}$ and partially suppressed deeper in the sample according to:

$$
\psi(0, z)= \begin{cases}0 & , \mathcal{F}(z)>\mathcal{F}_{\mathrm{T}} \\ \left(1-\frac{\mathcal{F}}{\mathcal{F}_{\mathrm{T}}} e^{-z / \lambda}\right) \sqrt{1-\frac{T_{1}(0, z)}{T_{\mathrm{c}}}} & , \mathcal{F}(z)<\mathcal{F}_{\mathrm{T}} .\end{cases}
$$

This expression can be considered as the limiting case of the fast quench without fluctuations.

The solution of the TDGL equations then corresponds to the propagation of the $S \rightarrow N$ boundary towards the surface in the form of a $S / N$ soliton wall. The recovery of the system is completely determined by the soliton propagation with only one free fit parameter, $\tau_{\mathrm{GL}}$, defining the velocity of the soliton $v_{\psi} \sim \xi_{\perp} / \tau_{\mathrm{GL}}[24,49]$. By setting $\tau_{\mathrm{GL}}=50 \mathrm{fs}$ we obtain the recovery on the experimentally observed timescale. However, the obtained trajectories $A_{\mathrm{S}}\left(\Delta t_{\mathrm{D}-\mathrm{P}}\right)$ are 
much sharper than experimentally observed [Fig. 3(b)]. For the weakest excitation $\mathcal{F}(z=0)=4.2 \mu \mathrm{J} / \mathrm{cm}^{2}$ the condensate is destroyed only at the surface and the modeled recovery occurs very rapidly within $50 \mathrm{fs}$. However, for the strongest excitation $\mathcal{F}=34 \mu \mathrm{J} / \mathrm{cm}^{2}$ the slow quench condition is satisfied and the $\mathrm{S} / \mathrm{N}$ boundary adiabatically follows the temperature front. In this case the simulated curve fits reasonably well to the data [Fig. 3(b)]. Nevertheless, we see that this second approach still fails to consistently describe the recovery at all destruction fluences. The gradual growth of the $S$ signal on the $10 \mathrm{ps}$ timescale for the intermediate fluences might be reproduced if one assumes that the soliton propagation is slow enough and the superconductivity emerges from a seed-being either preformed pairs or superconducting fluctuations.

In a simple preformed pair scenario superconductivity arises from the paired pseudogap-forming carriers. The density of these carriers provides the initial conditions for solving the TDGL equation. From previous experiments we know that in LSCO the pseudogap response is linear with fluence up to $\mathcal{F}_{\text {PG }} \sim 750 \pm 200 \mu \mathrm{J} / \mathrm{cm}^{2}$ [33]. At these fluences the material undergoes a structural instability [50], which naturally affects the response [33]. We can take $\mathcal{F}_{\mathrm{PG}}$ as the lower estimate for the fluence at which all pseudogap forming carriers are excited (or in the preformed pairs terms all pairs are depaired). This implies that at excitation conditions considered in this work $\mathcal{F} \leqslant 34 \mu \mathrm{J} / \mathrm{cm}^{2}$ the pseudogap carrier density would be suppressed at most by $5 \%$ (confirmed also by the robustness of the PG response in the present three-pulse experiments). The initial conditions for Eq. (3) would therefore vary with the depth and fluence by less than 5\%. Such fluence-independent initial conditions are inconsistent with the fluence dependence of the data, so the PG-forming carriers do not appear to seed the $S$ order parameter.

\section{THE ROLE OF FLUCTUATIONS}

A more realistic scenario invokes the thermal fluctuations of the superconducting order above $T_{\mathrm{c}}$, which are responsible for the order formation within the Kibble-Zurek scenario. After the $S$ order has been destroyed and the temperature is still significantly above $T_{\mathrm{c}}\left(\Delta t_{\mathrm{D}-\mathrm{P}} \sim 0.5 \mathrm{ps}\right)$, weak shortlived superconducting fluctuations exist in the system. As the electronic subsystem rapidly cools the density of the $S$ fluctuations increases, and their lifetime and correlation length diverge when $T \rightarrow T_{\mathrm{c}}$. During the initial cooling stage $\left(T \gg T_{\mathrm{c}}\right.$ ) fluctuations are fast and adapt to variations of temperature. However, after a certain moment in time given by $\tau_{\mathrm{Z}}=\sqrt{\tau_{\mathrm{GL}} \tau_{\mathrm{q}}}$, their lifetime becomes larger than the quench time, meaning that the system crosses the transition in a "frozen" inhomogeneous configuration. As the system escapes the critical region $T<T_{\mathrm{c}}\left(1-\frac{\tau_{\mathrm{Z}}}{\tau_{\mathrm{q}}}\right)$ the fluctuation lifetime decreases and the system starts to adapt to the new conditions, i.e., the order parameter grows from fluctuations according to TDGL theory. The appropriate expression for the density of such fluctuations, which we can implement as the initial condition have been given by Volovik $[51,52]$

$$
\psi_{\text {fluc }}(0, z) \sim\left(\frac{\tau_{\mathrm{GL}}}{\tau_{\mathrm{q}}(z)}\right)^{3 / 8} \frac{T_{\mathrm{c}}}{E_{\mathrm{F}}} \psi_{\mathrm{eq}}\left(T_{1}\right) .
$$

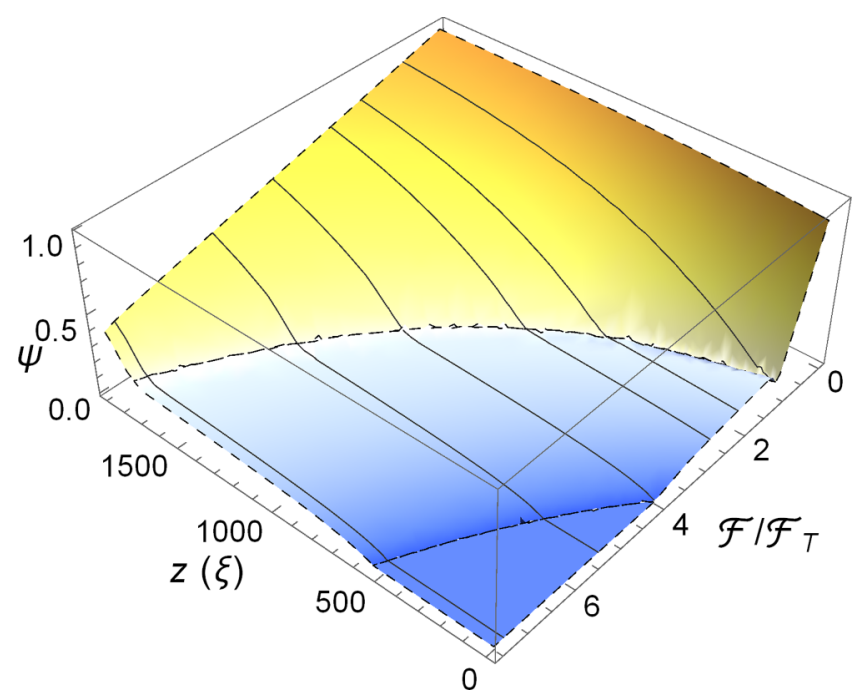

FIG. 5. The initial conditions that take into account fluctuations of the order parameter according to Eq. (4) (blue surface) and the partially suppressed (yellow surface) order parameter in the region $\mathcal{F}(z)<\mathcal{F}_{\mathrm{T}}$ in agreement with Eq. (3). The flat region near $z=0$ at $\mathcal{F} / \mathcal{F}_{\mathrm{T}}>4$ corresponds to the slow quench conditions.

The factor $\frac{T_{\mathrm{c}}}{E_{\mathrm{F}}}$ gives the correct order of magnitude $\sim 0.01$ of the seed order parameter to fit the data, whereas $\tau_{\mathrm{q}}$ and $\psi_{\text {eq }}\left(T_{1}\right)$ define the depth dependence. The spatial dependence of the initial order parameter obtained using (4) is shown in Fig. 5. We note that the initial temperature immediately after the optical energy absorption is not important because the properties of the seed OP are defined at $T_{\mathrm{Z}}=T_{\mathrm{c}}\left(1+\frac{\tau_{\mathbb{Z}}}{\tau_{\mathrm{q}}}\right)$. For the simulations we supplemented Eq. (4) with a proportionality factor $C: \psi_{\text {ini }}\left(\mathcal{F}(z)>\mathcal{F}_{\mathrm{T}}\right)=C \cdot \psi_{\text {fluc }}(0, z)$ which, in addition to $\tau_{\mathrm{GL}}$, was an adjustable parameter shared between all the curves. The resulting trajectories are shown in Fig. 3(c) (the parameter values are $\tau_{\mathrm{GL}}=1.25 \mathrm{ps}$ and $C=4$ ). Agreement between simulations and the experimental data is now much better.

Finally, we further improve the fit by solving the TDGL equation with parametrized phenomenological initial conditions which resemble the main feature of the Volovik's theory, i.e., growth of the fluctuations with depth. The goal here is to provide the initial conditions where the depth dependence is adjustable rather than defined by the quench rate deduced from the 3TM, which may not be sufficiently accurate. Instead of Eq. (4) we use a minimal model which produces a good fit:

$$
\psi_{\text {fluc }}(0, z)=\kappa z / \lambda_{\mathrm{p}}
$$

where $\kappa$ is the fitting parameter independent for each fluence value. The result of these simulations is presented in Fig. 3(d) with $\kappa$ values equal to $0.25,0.23,0.162,0.13,0.1$, and 0.06 in the order of increasing fluence, and $\tau_{\mathrm{GL}}=1.1 \mathrm{ps}$. Note that the fluctuations amplitude is limited in depth by the N/S boundary in this model and reaches at most $18 \%$ of the equilibrium order parameter value. The good correspondence of the simulation to the data justifies the initial conditions within the fluctuation scenario and underlines the importance of the fluctuations especially for low and intermediate fluences. 
There are a few physical reasons why the Volovik's description of fluctuations, i.e., our third approach is not sufficient. Firstly, it relies on the quench rates extracted from the 3TM, which by itself might not be sufficiently accurate. Secondly, it has been widely discussed that the cuprates have extremely strong fluctuations compared to the conventional superconductors and their onset in certain families exceeds the critical temperature by several tens of $\mathrm{K}$ [41,53-56]. Thus a more sophisticated treatment is required than the simple Gaussian fluctuations assumed by Volovik. Stronger fluctuations in BSCCO might also explain why the recovery of the condensate occurs one order of magnitude faster [18] than in LSCO. In turn we expect that Eq. (4) should work much better in the case of conventional superconductors.

We should note that despite the main advantage of the LSCO system-its long-lasting recovery of the condensate-which allowed us to discriminate the trajectory from short living excitations, there is a significant factor which cannot be easily accounted in the TDGL equations: the short lifetime fluctuations of the stripe order [57]. Recent studies have shown that the stripe fluctuations lifetime is below 2 ps [58]. Thus their coherent dynamics will influence the emerging $\mathrm{S}$ order parameter mainly through the initial conditions. One might speculate that the stripe fluctuations make the fit worse with the use of initial conditions suggested by Volovik (4) in comparison to the phenomenological ones (5).

In the Supplemental Material [27] we show that the remaining discrepancy between the fit and the data near $\sim 10 \mathrm{ps}$ for the two fastest quench rates can be accounted for by the suppression of the order parameter due to vortex formation. From the discussion above one can see that in the case of a fast quench the ergodicity of the system is broken as soon as the fluctuation timescale becomes longer than the quench time. The system then cannot follow the time evolution of the potential and evolves inhomogeneously through the transition with the appearance of vortices, quite faithfully reproduced by the model when the dynamics of the superconducting phase is explicitly included in the calculations (Fig. 5 of the Supplemental Material [27]).

\section{CONCLUSIONS}

By analyzing systematic fluence dependent ultrafast laser quench experiments in a prototype high-temperature superconductor, we conclude that the recovery of the superconducting order after the nonequilibrium phase transition can be described rather well within the framework of the time-dependent Ginzburg Landau theory. The predicted recovery of the superconducting order agrees quite well with the experiments in a wide range of destruction fluences when fluctuations seeding the growth of the order parameter are taken into account. This emphasizes the crucial role of fluctuations for the time evolution of the order parameter through the $N \rightarrow S$ transition.

The processes leading to the fast, $\lesssim 1 \mathrm{ps}$, initial destruction of superconducting order are beyond the TDGL modeling. This is not surprising considering that the destruction proceeds under highly nonergodic conditions [12].

Remarkably, the experiments show that photoexcitation does not affect the transient signatures of the PG state, and the superconducting order appears to grow out of the nearly unperturbed pseudogap state. The strong fluence dependence of the superconducting order recovery, concurrent with the fluence-independent PG signatures, implies that the PG forming carriers do not seed the initial growth of the superconducting order.

\section{ACKNOWLEDGMENTS}

We wish to acknowledge the useful discussion with T. W. Kibble regarding the importance of a variable quench rate in the experiment. The funding was provided by European Research Council advanced grant TRAJECTORY.
[1] Y. M. Bunkov and H. Godfrin, in Topological Defects and the Non-Equilibrium Dynamics of Symmetry Breaking Phase Transitions, edited by Y. M. Bunkov and H. Godfrin (Springer Netherlands, Dordrecht, 2000), p. 396.

[2] P. W. Higgs, Phys. Rev. 145, 1156 (1966).

[3] G. E. Volovik, The Universe in a Helium Droplet, International Series of Monographs on Physics, Vol. 117 (Oxford University Press, Oxford, 2009), p. 534.

[4] C. M. Varma, J. Low Temp. Phys. 126, 901 (2002).

[5] T. W. B. Kibble and G. E. Volovik, J. Exp. Theor. Phys. Lett. 65, 102 (1997).

[6] W. Zurek, Phys. Rep. 276, 177 (1996).

[7] R. Monaco, J. Mygind, M. Aaroe, R. J. Rivers, and V. P. Koshelets, Phys. Rev. Lett. 96, 180604 (2006).

[8] D. Golubchik, E. Polturak, and G. Koren, Phys. Rev. Lett. 104, 247002 (2010).

[9] W. H. Zurek, Nature (London) 317, 505 (1985).

[10] A. Schmid and G. Schön, J. Low Temp. Phys. 20, 207 (1975).

[11] A. Schmid, Phys. Kondens. Mater. 5, 302 (1966).
[12] P. Kusar, V. V. Kabanov, S. Sugai, J. Demsar, T. Mertelj, and D. Mihailovic, Phys. Rev. Lett. 101, 227001 (2008).

[13] C. Giannetti, G. Coslovich, F. Cilento, G. Ferrini, H. Eisaki, N. Kaneko, M. Greven, and F. Parmigiani, Phys. Rev. B 79, 224502 (2009).

[14] G. Coslovich, C. Giannetti, F. Cilento, S. Dal Conte, G. Ferrini, P. Galinetto, M. Greven, H. Eisaki, M. Raichle, R. Liang, A. Damascelli, and F. Parmigiani, Phys. Rev. B 83, 064519 (2011).

[15] C. Gadermaier, A. S. Alexandrov, V. V. Kabanov, P. Kusar, T. Mertelj, X. Yao, C. Manzoni, D. Brida, G. Cerullo, and D. Mihailovic, Phys. Rev. Lett. 105, 257001 (2010).

[16] C. Giannetti, M. Capone, D. Fausti, M. Fabrizio, F. Parmigiani, and D. Mihailovic, arXiv:1601.07204 [cond-mat.supr-con].

[17] V. V. Kabanov, J. Demsar, and D. Mihailovic, Phys. Rev. Lett. 95, 147002 (2005).

[18] C. L. Smallwood, W. Zhang, T. L. Miller, C. Jozwiak, H. Eisaki, D.-H. Lee, and A. Lanzara, Phys. Rev. B 89, 115126 (2014).

[19] G. P. Segre, N. Gedik, J. Orenstein, D. A. Bonn, R. Liang, and W. N. Hardy, Phys. Rev. Lett. 88, 137001 (2002). 
[20] N. Gedik, J. Orenstein, R. Liang, D. a. Bonn, and W. N. Hardy, Science (New York, NY) 300, 1410 (2003).

[21] A. Pashkin, M. Porer, M. Beyer, K. W. Kim, A. Dubroka, C. Bernhard, X. Yao, Y. Dagan, R. Hackl, A. Erb, J. Demsar, R. Huber, and A. Leitenstorfer, Phys. Rev. Lett. 105, 067001 (2010).

[22] M. Beyer, D. Städter, M. Beck, H. Schäfer, V. V. Kabanov, G. Logvenov, I. Bozovic, G. Koren, and J. Demsar, Phys. Rev. B 83, 214515 (2011).

[23] L. Perfetti, P. A. Loukakos, M. Lisowski, U. Bovensiepen, H. Eisaki, and M. Wolf, Phys. Rev. Lett. 99, 197001 (2007).

[24] The 1D time dependent GL equation $\tau_{\mathrm{GL}} \dot{\psi}=\xi^{2} \psi^{\prime \prime}+$ $\left(1-T / T_{\mathrm{c}}\right) \psi-\psi^{3}$ has a propagating soliton solution $\psi \propto$ $\tanh \left[\left(v_{\psi} t-z\right) / w\right]-1$ where $w=2 \sqrt{2} \xi / \sqrt{1-T / T_{\mathrm{c}}}$ and $v_{\psi}=3 \xi \sqrt{1-T / T_{\mathrm{c}}} / \sqrt{2} \tau_{\mathrm{GL}}$. Taking $\xi \sim \xi_{\perp} \sim 0.2 \mathrm{~nm}$ and $\tau_{\mathrm{GL}} \sim 100$ fs gives $v_{\psi}<5 \mathrm{~nm} / \mathrm{ps}$.

[25] I. S. Aranson, N. B. Kopnin, and V. M. Vinokur, Phys. Rev. Lett. 83, 2600 (1999).

[26] R. Yusupov, T. Mertelj, V. V. Kabanov, S. Brazovskii, P. Kusar, J.-H. Chu, I. R. Fisher, and D. Mihailovic, Nat. Phys. 6, 681 (2010).

[27] See Supplemental Material at http://link.aps.org/supplemental/ 10.1103/PhysRevB.93.224520 for the derivation of the response function, three pulse data simulation, and solution of the complete set of TDGL equations.

[28] Y. H. Liu, Y. Toda, K. Shimatake, N. Momono, M. Oda, and M. Ido, Phys. Rev. Lett. 101, 137003 (2008).

[29] C. Giannetti, F. Cilento, S. D. Conte, G. Coslovich, G. Ferrini, H. Molegraaf, M. Raichle, R. Liang, H. Eisaki, M. Greven, A. Damascelli, D. van der Marel, and F. Parmigiani, Nat. Commun. 2, 353 (2011).

[30] R. D. Averitt, G. Rodriguez, A. I. Lobad, J. L. W. Siders, S. A. Trugman, and A. J. Taylor, Phys. Rev. B 63, 140502 (2001).

[31] R. D. Averitt and A. J. Taylor, J. Phys.: Condens. Matter 14, R1357 (2002).

[32] P. Kusar, J. Demsar, D. Mihailovic, and S. Sugai, Phys. Rev. B 72, 014544 (2005).

[33] P. Kusar, V. V. Kabanov, S. Sugai, J. Demšar, T. Mertelj, and D. Mihailović, J. Supercond. Novel Magn. 24, 421 (2010).

[34] V. V. Kabanov, J. Demsar, B. Podobnik, and D. Mihailovic, Phys. Rev. B 59, 1497 (1999).

[35] J. Demsar, R. Hudej, J. Karpinski, V. Kabanov, and D. Mihailovic, Physica C 341-348, 925 (2000).

[36] R. a. Kaindl, Science 287, 470 (2000).

[37] G. Coslovich, C. Giannetti, F. Cilento, S. Dal Conte, T. Abebaw, D. Bossini, G. Ferrini, H. Eisaki, M. Greven, A. Damascelli, and F. Parmigiani, Phys. Rev. Lett. 110, 107003 (2013).
[38] Y. Toda, F. Kawanokami, T. Kurosawa, M. Oda, I. Madan, T. Mertelj, V. V. Kabanov, and D. Mihailovic, Phys. Rev. B 90, 094513 (2014).

[39] For maximum accuracy we subtract the signal at $\Delta t_{\mathrm{D}-\mathrm{P}}=0.2 \mathrm{ps}$ after the $D$ pulse at $34 \mathrm{~K}\left(6 \mathrm{~K}\right.$ above $\left.T_{\mathrm{c}}\right)$. This allows to completely separate the pseudogap from the superconducting signal and give a good estimate for the remaining signal at timescales up to $1 \mathrm{ps}$.

[40] I. K. Schuller and K. E. Gray, J. Supercond. Novel Magn. 19, 401 (2006).

[41] I. Madan, T. Kurosawa, Y. Toda, M. Oda, T. Mertelj, P. Kusar, and D. Mihailovic, Sci. Rep. 4, 5656 (2014).

[42] L. Gor'kov and N. Kopnin, Usp. Fiz. Nauk 116, 413 (1975).

[43] M. Suzuki and M. Hikita, Phys. Rev. B 44, 249 (1991).

[44] B. Mansart, D. Boschetto, A. Savoia, F. Rullier-Albenque, F. Bouquet, E. Papalazarou, A. Forget, D. Colson, A. Rousse, and M. Marsi, Phys. Rev. B 82, 024513 (2010).

[45] H.-H. Wen, Z.-Y. Liu, F. Zhou, J. Xiong, W. Ti, T. Xiang, S. Komiya, X. Sun, and Y. Ando, Phys. Rev. B 70, 214505 (2004).

[46] A. Junod, A. Bezinge, D. Cattani, J. Cors, M. Decroux, $\varnothing$. Fischer, P. Genoud, L. Hoffmann, J.-L. Jorda, J. Muller, and E. Walker, Jpn. J. Appl. Phys. 26, 1119 (1987).

[47] L. Stojchevska, P. Kusar, T. Mertelj, V. V. Kabanov, Y. Toda, X. Yao, and D. Mihailovic, Phys. Rev. B 84, 180507 (2011).

[48] V. V. Baranov and V. V. Kabanov, Phys. Rev. B 89, 125102 (2014).

[49] $\xi_{\perp}$ is a constant obtained from independent experiments.

[50] N. Gedik, D.-S. Yang, G. Logvenov, I. Bozovic, and A. H. Zewail, Science 316, 425 (2007).

[51] G. E. Volovik, Physica B 280, 122 (2000).

[52] In the original paper the misprint occurred: The power of the fraction was $1 / 8$ instead of $3 / 8$.

[53] Z. Xu, N. Ong, Y. Wang, T. Kakeshita, and S. Uchida, Nature (London) 406, 486 (2000).

[54] A. Junod, M. Roulin, B. Revaz, and A. Erb, Physica B 280, 214 (2000).

[55] J. Corson, R. Mallozzi, and J. Orenstein, Nature (London) 398 , 221 (1999).

[56] L. Perfetti, B. Sciolla, G. Biroli, C. J. van der Beek, C. Piovera, M. Wolf, and T. Kampfrath, Phys. Rev. Lett. 114, 067003 (2015).

[57] S. Sugai, Y. Takayanagi, and N. Hayamizu, Phys. Rev. Lett. 96, 137003 (2006).

[58] D. H. Torchinsky, F. Mahmood, A. T. Bollinger, I. Božović, and N. Gedik, Nat. Mater. 12, 387 (2013). 